\title{
A modified endaural approach to the fractured condyle: a clinical case report
}

\author{
Laís Inês Silva Cardoso 1, Ana Caroline Ramos de Brito 2, Simei André Rodrigues da Silva Freire 3, \\ Paulo Henrique Luiz de Freitas 4, Renato da Costa Ribeiro 3
}

\begin{abstract}
${ }^{1}$ Resident of Oral and Maxillofacial Surgery, University Hospital of Federal University of Piaui, Piaui, Brazil.
2 Department of Dentistry, Federal University of Piaui, Brazil.

${ }^{3}$ Oral and Maxillofacial Surgery, University Hospital of Federal University of Piaui, Piaui, Brazil.

${ }^{4}$ Department of Dentistry, Federal University of Sergipe at Lagarto, Sergipe, Brazil.
\end{abstract}

${ }^{*}$ Corresponding author: Laís Inês Silva Cardoso. University Hospital, Federal University of Piauí. Campus Universitário Ministro Petrônio Portela, SG 07 s/n - Ininga, Teresina - PI, 64049-550 E-mail: laisinescardoso@gmail.com.

Research Ethics Committee Approval (if necessary): We declare that the patient approved the study by signing an informed consent form and the study followed the ethical guidelines established by the Declaration of Helsinki.

Received on: Nov 26, 2021. Accepted on: Dec 16, 2021. Available online: Dec 21, 2021.

\section{Abstract}

The authors present a modified endaural incision with a caudal extension for surgical access to the mandibular condyle. The approach combined the advantages of good scar cosmesis and sufficient access to the surgical site for optimal placement of the osteosynthesis with lesser risk of nerve damage.

Keywords: Bone Fractures; Mandibular Condyle; Facial Nerve Injuries; Minimally Invasive Surgical Procedures.

\section{Introduction}

Choosing between open and closed management of fractures of the mandibular condyle is a topic far from consensual. The main concern of a surgeon faced with that choice is the possibility of facial nerve injury. However, improvements in surgical technique and diagnostic tools, along with better understanding of local anatomy and the availability of internal fixation devices, led to conclusions that surgical treatment may produce better results when compared to conservative treatment - at least in certain cases [1].

As professionals dealing with the surgical handling of hard and soft tissues of the human face, oral and maxillofacial (OMF) surgeons are especially careful with incision placement and try to restore function without compromising facial esthetics. Thus, we present a modified endaural incision with a caudal extension to treat a mandibular condylar fracture with 
sufficient access to 40 the bone fragments but with better cosmesis if compared to placement of a retromandibular approach.

\section{Case report}

A 25 years-old female victim of a motorcycle accident was admitted to the hospital and evaluated by OMF Surgery staff after being considered medically stable. Her chief complains were occlusal alteration and pain on palpation of the right preauricular region. Upon clinical and tomographic examination, a condylar neck fracture with anteromedial dislocation and an incomplete mandibular symphysis fracture were identified (Figure 1A and 1B).

Surgical treatment consisted of reduction and fixation of fractures under general anesthesia. The incision started at the internal face of the tragus and proceeded inferiorly around the ear lobe. Then, a lower extension of approximately $10 \mathrm{~mm}$ was performed along the posterior border of the mandibular ramus through the skin and subcutaneous tissues (Figure 1C).

After retraction of the most superficial tissues, the platysma and the superficial musculoaponeurotic system (SMAS) were cut parallel to the posterior border of the mandibular ramus. As soon as glandular tissue from the parotid was seen, we dissected bluntly in an anteromedial direction towards the posterior edge of the ramus. At that point, some branches of the facial nerve were found and dissected anteriorly and posteriorly by approximately $10 \mathrm{~mm}$ and $5 \mathrm{~mm}$, respectively, thus allowing for tissue retraction with minimal tension. In a deeper plane, we found the masseter close to the pterigomasseteric sling, with the retromandibular vein out of sight and protected beneath the parotid gland.

The sling was incised at the posterior border of the ramus and periosteum elevators were used for exposing the fracture site. To 61 expedite fracture reduction, we placed a screw at the mandibular angle then tied it to a wire that was passed transcutaneously to pull the mandible in a caudal direction. Once the fracture was reduced, maxillomandibular fixation was applied and internal fixation achieved with two titanium miniplates, one positioned on the posterior edge and the other positioned on the lateral region of the mandibular ramus (Figure 2A and 2B).

Occlusion and mandibular mobility were checked, and the surgical wound was subsequently closed in layers. One year after surgery, the patient was asymptomatic and pleased with the esthetic and functional results. Occlusion and mandibular movements were preserved, without deviations on mouth opening. Facial nerve function was unaltered, and the surgical scar was practically imperceptible, hidden behind the earlobe (Figure 2C). 

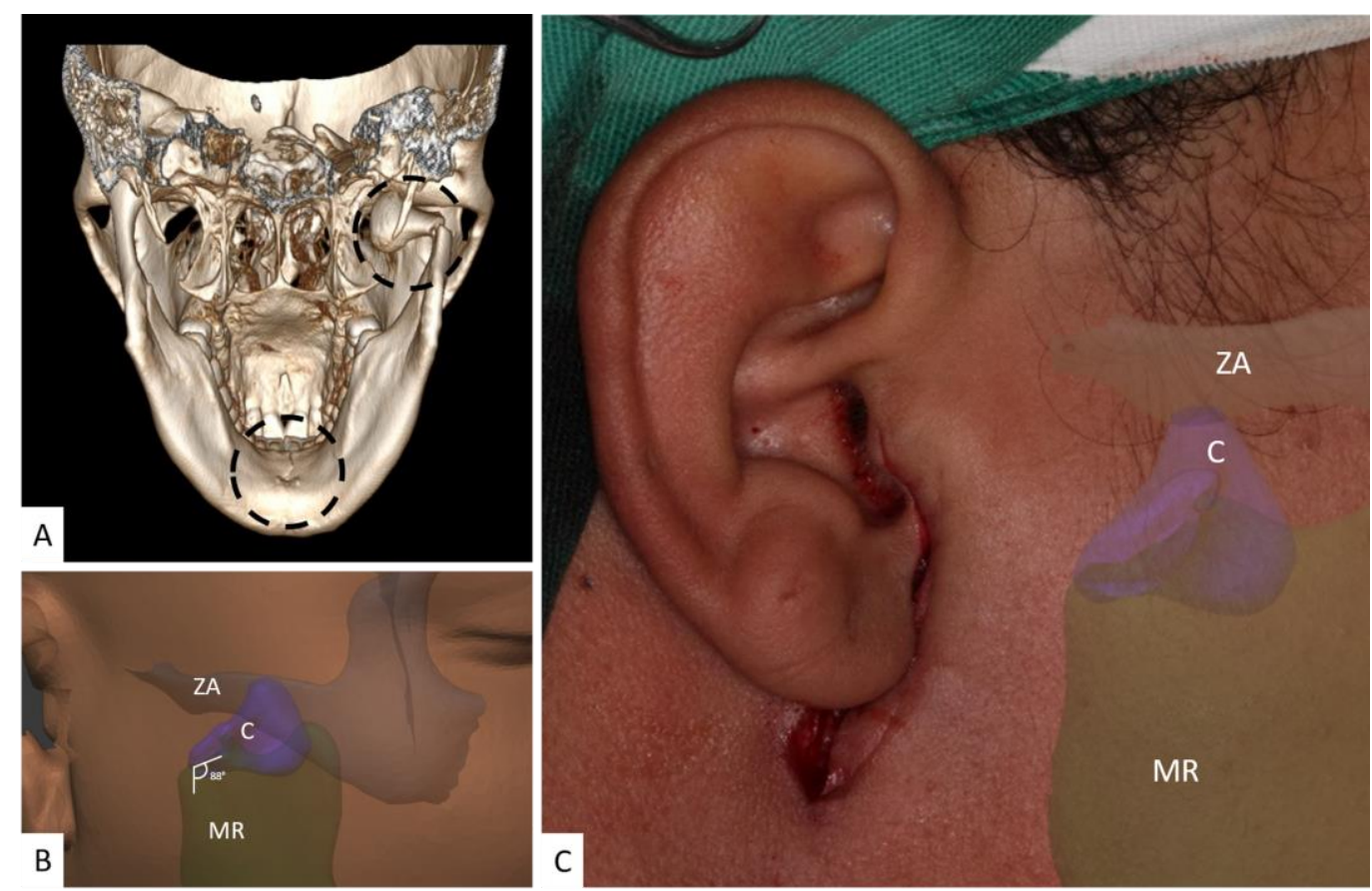

Figure 1: A, Tomography-based three-dimensional (3D) reconstruction from a posteroanterior view showing the dislocated right condylar fracture and the incomplete symphyseal fracture. B, Tomography-based, artificially colored 3D reconstruction illustrating the degree of fracture dislocation ( $88^{\circ}$ between the fragments). C, Actual endaural incision with inferior extension and superimposed anatomic structures for spatial reference. ZA, zygomatic arch; C, condyle; MR, mandibular ramus.
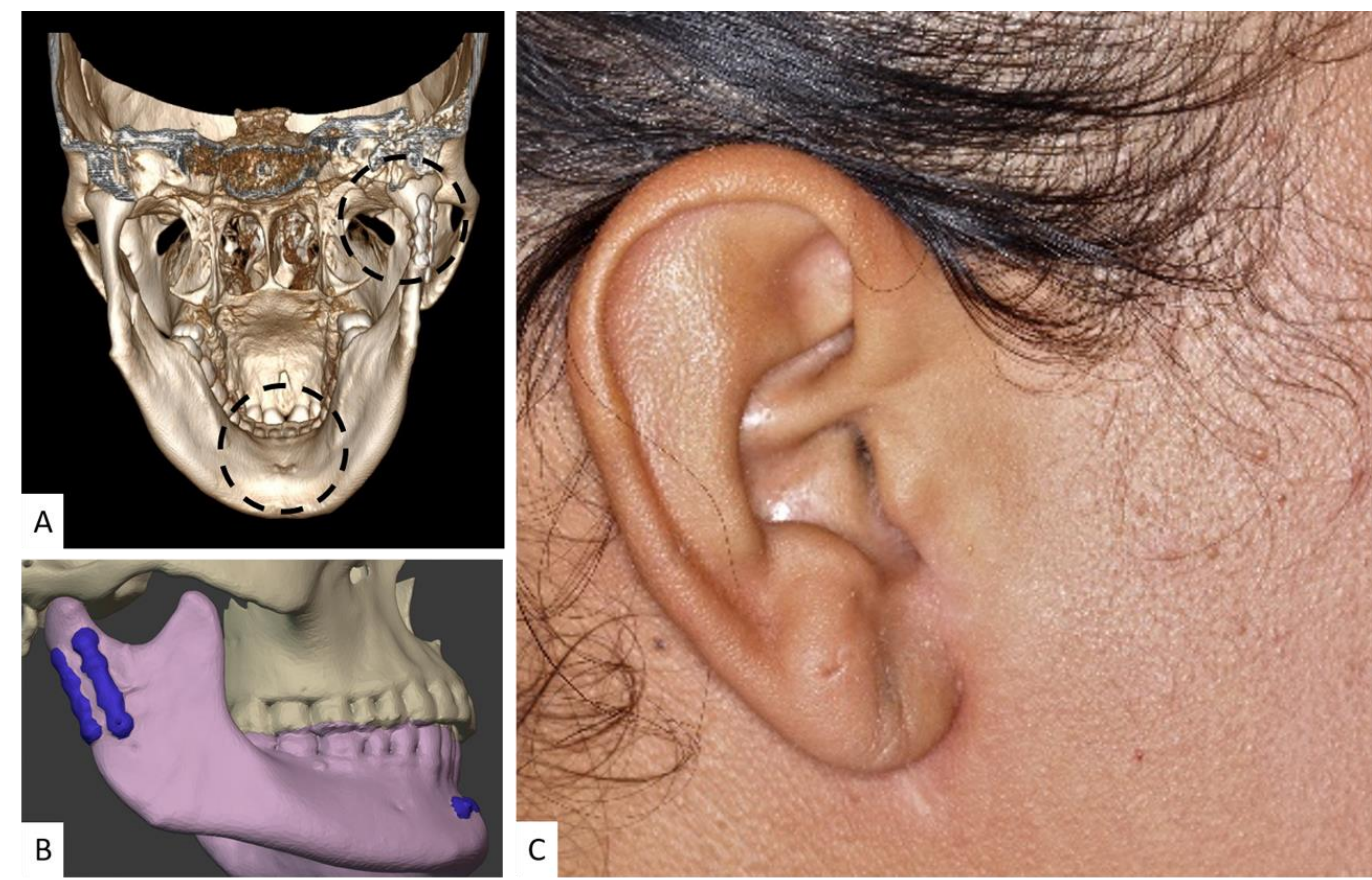

Figure 2: A, Tomography-based three-dimensional (3D) reconstruction from a posteroanterior view showing the right condylar and the symphyseal fracture sites one year after open treatment (traced black circles). B, Tomography-based, artificially colored 3D reconstruction showing the adequate condyle-fossa relation and the stable occlusion. C, Discrete surgical scar one year after treatment. 


\section{Discussion and Conclusion}

Open reduction and internal fixation of condyle fractures has been regarded as the most effective treatment for condylar fractures with deviations greater than 45 degrees [2]. In the present case, the condylar fragment was bent $88^{\circ}$ medially, outside the glenoid fossa.

After surgery, the patient evolved with short-term facial nerve palsy, but with complete recovery after 1 year. Mandibular excursions were unhindered, painless, silent and non-deviated, and the patient was pleased with the cosmesis of the surgical scar. Indeed, in their meta analyzes, Al-Moraissi et al. [3] and Chrcanovic [4] concluded that the results of open treatment for condylar fractures show better outcomes in terms of mandibular mobility, chin deviation and malocclusion, findings that are compatible with those found in this case.

If one takes the periauricular approach for surgical facelift described by Vesnaver et al. [5] and the one proposed here, it is safe to say that both allow for good inspection, reduction and fixation of all condylar fractures ranging from high, dislocated condylar fractures to lower ramus fractures. However, the first approach requires a longer incision, with greater need for tissue divulsion and an increased risk of great auricular nerve damage. On the other hand, the risk of injury to that nerve is greatly reduced with the approach presented in this paper with the added advantage of a shorter incision in the parotideomasseteric fascia, thus reducing the risk of postoperative salivary fistulae.

Still, a common advantage of both approaches is the easiness with which the screws can be inserted perpendicular to the bone surface, a crucial aspect for the stability of the condylar osteosynthesis. Balaji [6] presented a study with 75 patients with condyle fractures, treated with open reduction and internal fixation also by a modified approach, presenting a 92\% rate of success. Of these patients, twenty had bilateral condyle fractures, three present restricted mouth opening, and seven presented transient facial weakness. The surgical approach suggested by the author is more extensive than ours, associating with the Al-Kayat and Bramley technique.

Thus, we described an alternative surgical approach for mandibular condyle fractures that combines the advantages of good scar cosmesis and sufficient access to the surgical site for optimal placement of the osteosynthesis with lesser risk of nerve damage. Clinical trials in centres with larger case numbers and variety would help to validate the assumptions here raised. 


\section{References}

[1] Alyahya A, Bin Ahmed A, Nusair Y, Ababtain R, Alhussain A, Alshafei A. Mandibular condylar fracture: a systematic review of systematic reviews and a proposed algorithm for management [published online ahead of print, 2020 Mar 31]. Br J Oral Maxillofac Surg. 2020; S0266-4356(20):30099-1. doi: 10.1016/j.bjoms.2020.03.014.

[2] Vincent AG, Ducic Y, Kellman R. Fractures of the Mandibular Condyle. Facial Plast Surg. 2019; 35(6): 623-626. doi: 10.1055/s-0039-1700888.

[3] Al-Moraissi EA, Ellis E 3rd. Surgical treatment of adult mandibular condylar fractures provides better outcomes than closed treatment: a systematic review and meta-analysis. J Oral Maxillofac Surg. 2015; 73(3): 482-493. doi: 10.1016/j.joms.2014.09.027.

[4] Chrcanovic BR. Surgical versus non-surgical treatment of mandibular condylar fractures: a meta-analysis. Int J Oral Maxillofac Surg. 2015;44(2): 158179. doi:10.1016/j.ijom.2014.09.024. 108.

[5] Vesnaver A, Gorjanc M, Eberlinc A, Dovsak DA, Kansky AA. The periauricular transparotid approach for open reduction and internal fixation of condylar fractures. J Craniomaxillofac Surg. 2005; 33(3): 169-179. doi: 10.1016/j.jcms.2005.01.008
[6] Balaji SM. Modified endaural approach for the treatment of condylar fractures: A review of 75 cases. Indian J Dent Res. 2016: 27(3);305-311. doi: 10.4103/0970-9290.186235.

Conflict of interest: The author declares no conflicts of interest.

Funding: The author declares no funding to develop this article.

How to cite this article: Cardoso LIS, de Brito ACR, Freire SARS, Freitas PHL, Ribeiro RC. A modified endaural approach to the fractured condyle: a clinical case report. Brazilian Journal of Case Reports. 2022Jan-Mar;02(1):10-14. 